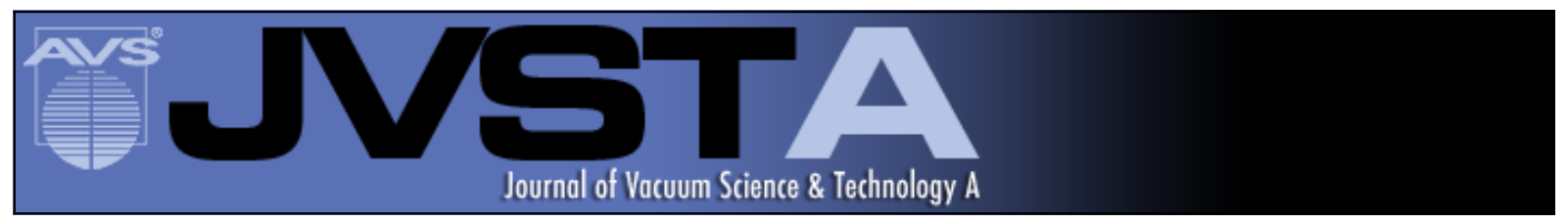

\title{
Study of a micro chamber quadrupole mass spectrometer
}

Jinchan Wang, Xiaobing Zhang, Fuming Mao, Mei Xiao, Yunkang Cui, Daniel den Engelsen, and Wei Lei

Citation: Journal of Vacuum Science \& Technology A 26, 239 (2008); doi: 10.1116/1.2827512

View online: http://dx.doi.org/10.1116/1.2827512

View Table of Contents: http://scitation.aip.org/content/avs/journal/jvsta/26/2?ver=pdfcov

Published by the AVS: Science \& Technology of Materials, Interfaces, and Processing

\section{Re-register for Table of Content Alerts}

\section{Create a profile. $\quad$ Sign up today!}

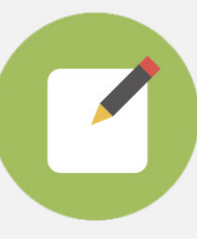




\title{
Study of a micro chamber quadrupole mass spectrometer
}

\author{
Jinchan Wang, Xiaobing Zhang, ${ }^{\text {a) }}$ Fuming Mao, Mei Xiao, Yunkang Cui, \\ Daniel den Engelsen, and Wei Lei \\ Jiangsu Information Display Engineering Research Center, School of Electronic Science and Engineering, \\ Southeast University, Nanjing 210096, People's Republic of China
}

(Received 23 July 2007; accepted 27 November 2007; published 29 January 2008)

\begin{abstract}
The design of a micro chamber quadrupole mass spectrometer (MCQMS) having a small total volume of only $20 \mathrm{~cm}^{3}$, including Faraday cup ion detector and ion source, is described. This MCQMS can resist a vacuum baking temperature of $400-500{ }^{\circ} \mathrm{C}$. The quadrupole elements with a hyperbolic surface are made of a ceramic material and coated with a thin metal layer. The quadrupole mass filter has a field radius of $3 \mathrm{~mm}$ and a length of $100 \mathrm{~mm}$. Prototypes of this new MCQMS can detect a minimum partial pressure of $10^{-8} \mathrm{~Pa}$, have a peak width of $\Delta M=1$ at $10 \%$ peak height from mass number 1 to 60, and show an excellent long-term stability. The new MCQMS is intended to be used in residual gas analyses of electron devices during a mutual pumping and baking process. (C) 2008 American Vacuum Society. [DOI: 10.1116/1.2827512]
\end{abstract}

\section{INTRODUCTION}

Mass spectrometers have been widely used as standard analytical instruments capable of providing qualitative and quantitative analysis of the elemental, chemical, and isotopic composition of gases. Different types of mass spectrometers have been developed for different application areas. However, they have disadvantages, such as relatively large size and complex maintenance, so further improvements are needed for the above considerations and particular application requirements of mass spectrometers. During the last few years, a growing interest has been shown in the miniaturization of mass spectrometry equipment, such as time-of-flight spectrometers, ${ }^{1}$ ion traps, ${ }^{2}$ sector mass filter, $^{3}$ and quadrupoles. ${ }^{4-6}$ Most of this miniaturization has been focused on the geometry of the mass analyzer, such as a miniature QMS and a simplified ion trap called a rectilinear ion trap $(\text { RIT) })^{2}$ with an internal volume of only $3.5 \mathrm{~cm}^{3}$. In many cases, it is not the mass analyzer itself but the associated vacuum and electronic components that limit the development of an entirely miniaturized instrument. ${ }^{6}$ The internal volume of the mass analyzer may be shrunken by reducing the size of the analyzer, but electronic components and fixing parts are still needed to be mounted in the vacuum shell for proper working of this small analyzer. So, a vacuum chamber larger than the mass analyzer is necessary for a miniaturized analyzer such as the RIT and miniaturized linear quadrupole mass spectrometer $(\mathrm{QMS}){ }^{7-11}$

Quadrupole mass spectrometers ${ }^{12}$ with cylindrical rods are commonly used and are very successful as mass filters because of their excellent performance and low cost. ${ }^{13}$ In the last 40 years, the most important progress in QMS technology was in the field of manufacturing and application in new measuring methods, notably combining high resolution organic chromatography with QMS and using miniature QMS for gas analysis (RGA) at relative high pressure.

\footnotetext{
${ }^{a}$ Author to whom correspondence should be addressed. Electronic mail: bell@seu.edu.cn
}

RGA of vacuum tubes is important in investigations of cathode properties. In these measurements, a sampling device is used to open the vacuum tubes leading the residual gas to the analysis chamber. A vacuum system for RGA with a traditional QMS may have a typical volume of 21 . If one wants to measure the residual gas composition of a small electron device having a volume of $0.01-0.11$, the gas will be diluted by a factor of 200 when being introduced into the analysis chamber. Generally, the pressure of an electron device is at a level of $10^{-6}-10^{-3} \mathrm{~Pa}$, so the influence from background gases of the analysis chamber and the opening tool plays a dominant role in the case of a small vacuum device. This will seriously affect the detection limit and the accuracy of the measurement.

If the volume of the QMS can be reduced, the adsorption and desorption surface area will be reduced at the same time. Therefore, it would be ideal to make the QMS as an annex of the (small) vacuum tube. After the mutual pumping and baking-out process together with the vacuum tube, the QMS can be operated to measure the residual gas in the chamber of the vacuum tube and QMS. Since the residual gas inside the vacuum tube is not seriously affected during this measurement, it may be repeated. After these measurements the QMS can be separated from the vacuum tube without influencing the vacuum performance of the measured device. This "mutual application" avoids the disadvantage of RGA of vacuum tubes by the traditional method.

Two kinds of miniature QMS have been reported up to the present, viz. by Ferran ${ }^{3-5}$ and Taylor, ${ }^{6,7}$ respectively, whose main objectives were to reduce the size of the quadrupoles to $0.5-1.0 \mathrm{~mm}$ in diameter and $10-30 \mathrm{~mm}$ in length. The application of miniature quadrupole rods degrades the resolution, sensitivity, and stability of sensitivity. Furthermore, these miniature QMSs cannot resist the $400-500{ }^{\circ} \mathrm{C}$ vacuum baking temperature of electron devices, so they do not meet the requirement of mutual pumping and baking.

In this paper we describe a micro chamber QMS (MCQMS). Our design objective is to develop a QMS with only 


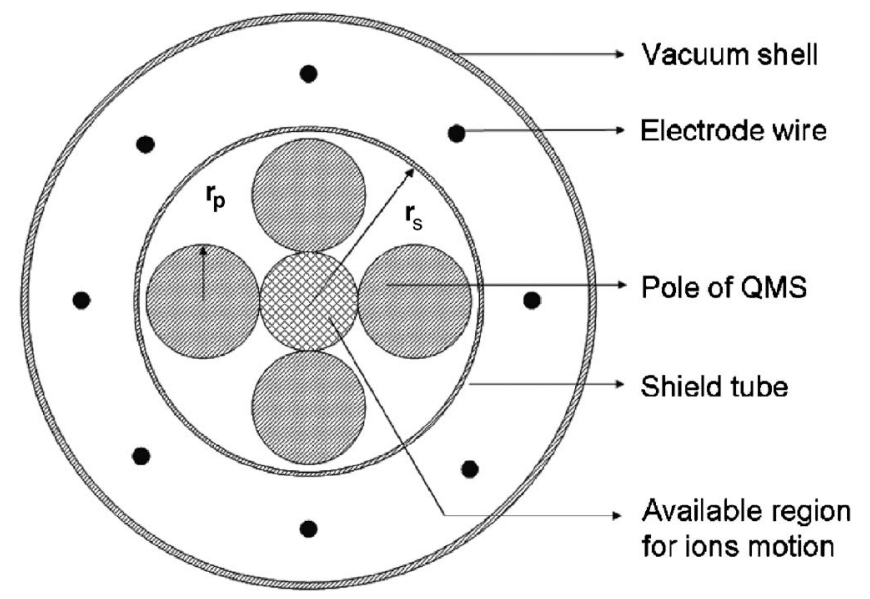

FIG. 1. Cross section of a conventional QMS.

the central area inside the $Q$-rods as vacuum chamber, that is, to keep the available space for ion motion inside vacuum whereas other components, such as electron wires and the mechanical fixing system of the $Q$-poles of the QMS, are not in the vacuum chamber. In this way, the vacuum volume can be largely reduced while maintaining the excellent performance of a conventional QMS.

\section{WORKING PRINCIPLE OF THE MCQMS}

The cross section of a commercial QMS is shown in Fig. 1. Ions move in the central circle area; the large outer volume contains the electrode wires, the mechanical fixing of the $Q$-poles, and the vacuum shielding tube. However, for the basic function of the QMS this large outer volume does not need to be kept in vacuum. The mass filter of a commercial QMS in Fig. 1 is composed of four metal poles. The central symmetry axis of the quadrupoles is called the field axis. The distance from this field axis to the quadrupoles is called the field radius $\left(r_{0}\right)$. To achieve a good hyperbolic electric field, the following relations should hold for QMS with cylindrical quadrupoles:

$$
\begin{aligned}
& r_{s}=3.54 r_{0}, \\
& r_{p}=1.1468 r_{0} .
\end{aligned}
$$

In the above equations $r_{s}$ is the radius of the inner shield tube and $r_{p}$ is the radius of the quadrupoles, as indicated in Fig. 1.

A typical commercial QMS for RGA has quadrupoles 6 $\mathrm{mm}$ in diameter and $100 \mathrm{~mm}$ in length. The inner diameter of the vacuum shell is $50 \mathrm{~mm}$, so the volume inside $r_{0}$ is only $1.1 \%$ of the total mass filter volume contained by the outer vacuum shell. If only the internal part of the mass analyzer is kept in vacuum while the large outer space can be moved out, the volume of the vacuum part will be greatly reduced. This consideration can also be used for the miniaturization of other types of mass spectrometer.

Figure 2 shows the cross section of our micro chamber mass filter, which has a field radius of $3 \mathrm{~mm}$ and a length of $100 \mathrm{~mm}$. This micro chamber mass filter has a total vacuum volume of only $8.4 \mathrm{~cm}^{3}$. Electrodes with a hyperbolic sur-

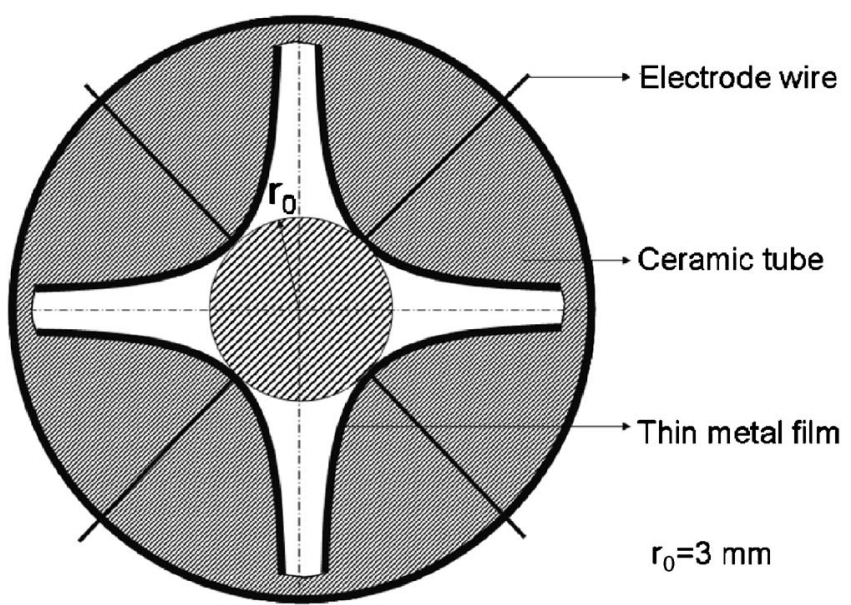

FIG. 2. Cross section of micro chamber QMS.

face are applied, generating the required $Q$-field very accurately in the MCQMS. The micro chamber mass filter, as shown in Fig. 3, is made of a ceramic material, viz. 95\% $\mathrm{Al}_{2} \mathrm{O}_{3}$, which is compatible with high vacuum requirements, i.e., low outgassing rate and being baked at $500{ }^{\circ} \mathrm{C}$ in vacuum. This filter is fabricated by means of die-casting and high temperature sintering without mechanical finishing techniques. After this, thin metal films are coated on the inner hyperbolic walls of the ceramic body. Between two adjacent segments of the filter the metal-film has been interrupted to enable good electrical insulation between these electrodes. Connection wires go through the ceramic in order not to disturb the electrical field and ion motion.

A photograph of the whole MCQMS is shown in Fig. 4. It is composed of an ion source housing and ion source vacuum chamber, ceramic mass filter, and Faraday cup ion detector. The ends of the ceramic mass filter are joined to the ion source and ion detector with a toroidal element made of kovar, which largely meets the expansion coefficient of alumina. The ion source for the MCQMS is of the electron collision type with double $\mathrm{Y}_{2} \mathrm{O}_{3}$ filaments as in a $B$ - $A$ gauge. Its vacuum chamber is $22 \mathrm{~mm}$ in diameter and $25 \mathrm{~mm}$ in length, making a volume of about $9.5 \mathrm{~cm}^{3}$. If a coaxial distributing ion source is used in the MCQMS, the vacuum chamber of the ion source could be further reduced to only

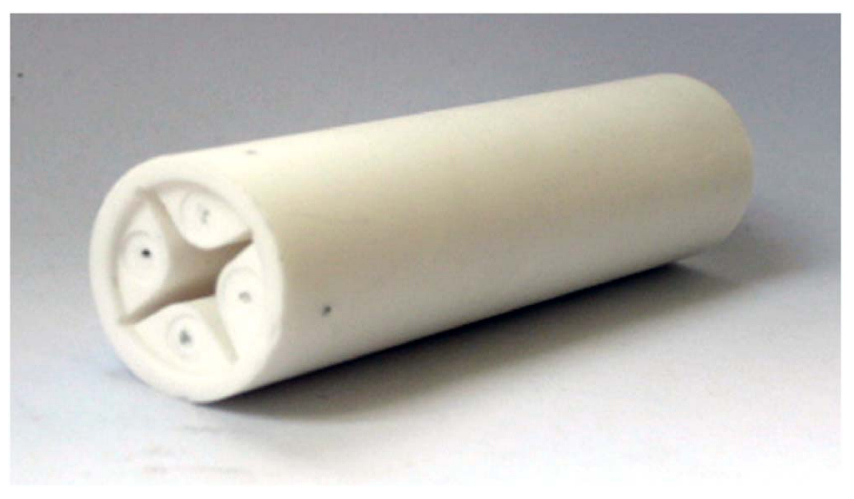

FIG. 3. Structure of MCQMS. 


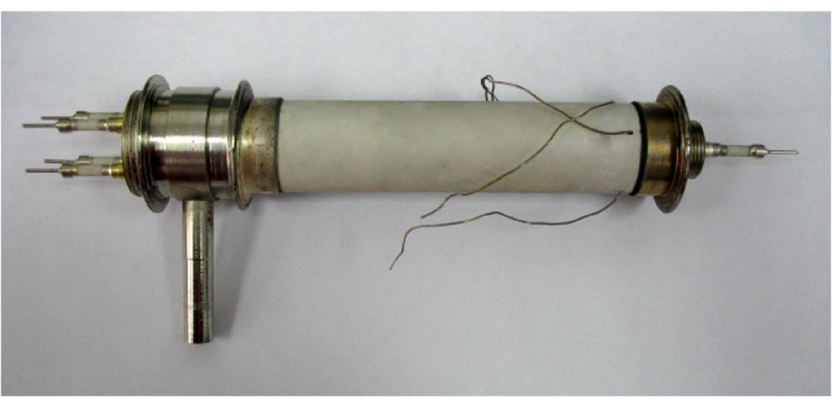

FIG. 4. Photograph of the MCQMS: (a) whole, (b) ceramic mass filter, and (c) ion source.

$5 \mathrm{~cm}^{3}$. The ion source is easy to clean and the filaments can be replaced as well. The frequency of the power supply for our MCQMS is about 4.2 MHz.

The main parameters of the MCQMS shown in Fig. 4 are as follows:

(1) vacuum volume of the MCQMS without connection tube is $20 \mathrm{~cm}^{3}$;

(2) mass filter has a field radius of $3 \mathrm{~mm}$ and a length of 100 $\mathrm{mm}$;

(3) mass range: 1-60 amu, resolution at 10\% peak height: $\Delta M<1$ amu;

(4) sensitivity of $10^{-6} \mathrm{~A} / \mathrm{Pa}$ with a Faraday cup ion detector;

(5) ionization electron current of the ion source is $I_{e}$ $=0-5 \mathrm{~mA}$;

(6) $\mathrm{rf}$ with a frequency of $f=4.2 \mathrm{MHz}$;

(7) minimum detectable partial pressure $P_{\min }=10^{-8} \mathrm{~Pa}$; and

(8) can be baked at $400-500{ }^{\circ} \mathrm{C}$ (in vacuum).

To validate whether the performance of our MCQMS design agrees with the target specification as mentioned in the previous section, a MCQMS was fabricated as shown in Fig. 4. It was connected to a UHV-analysis chamber of 0.51 for measurements. A sputter ion pump was applied to pump the vacuum system to UHV; a mechanical pump and a turbo molecular pump were used for initial pump down to the high vacuum range.

At a total pressure of about $1 \times 10^{-4} \mathrm{~Pa}$, measured with the UHV-gauge, the MCQMS was switched on and operated at an electron emission current of $1 \mathrm{~mA}$ and a scanning speed of $1 \mathrm{amu} / \mathrm{s}$. Figure 5 shows the typical mass spectra of the residual gases registered with the MCQMS: peaks of the residual gases inside the UHV-system were observed at 2, 18, 28 , and 44 amu. Peak shape and resolution meet the application requirement. Figure 5(b) is a magnification of the spectrum of Fig. 5(a) with a factor of 10 at the same driving conditions.

To estimate the performance of the MCQMS, we assume that the sensitivity of the MCQMS for different gases is the same. The pressure measured with the UHV-ionization gauge is indicated in $\mathrm{N}_{2}$ equivalent pressure.

The total ion current at this condition is about $1.5 \times 10^{-11}$ A calculated from Fig. 5(a) at a pressure of $1 \times 10^{-4}$ Pa. Peak 1 at mass 24 indicated in Fig. 5(b) corre-
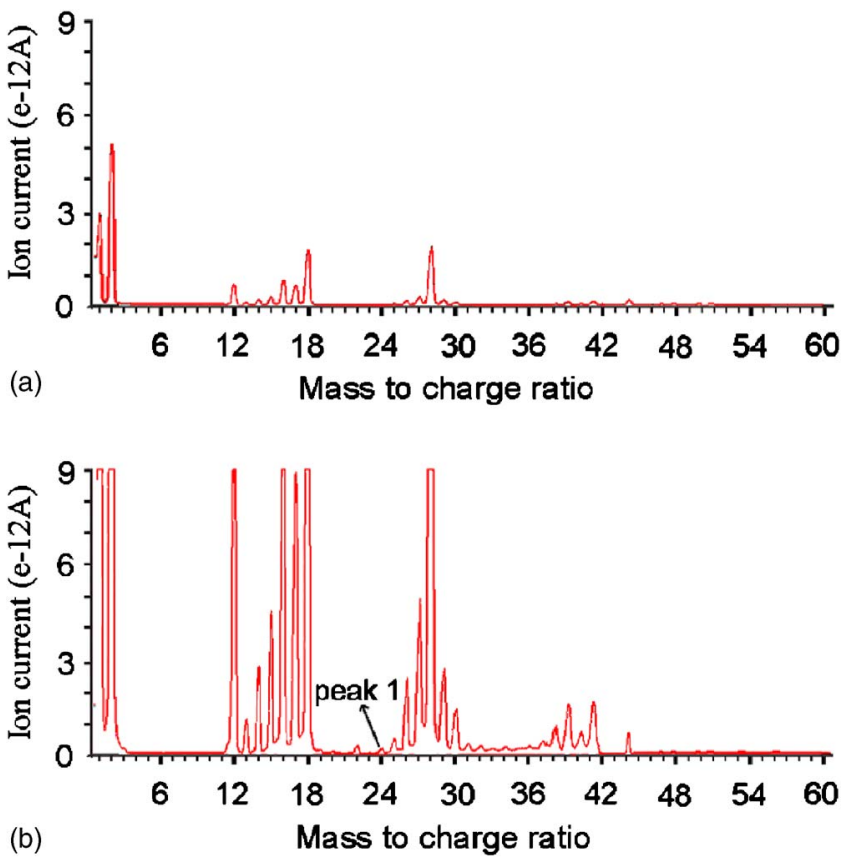

FIG. 5. Residual gas spectrum inside a UHV-system (base pressure 1.0 $\times 10^{-4} \mathrm{~Pa}$, electron emission current $1 \mathrm{~mA}$, scanning speed $\left.1 \mathrm{amu} / \mathrm{s}\right)$.

sponds to an ion current of $1 \times 10^{-14} \mathrm{~A}$ and represents a partial pressure of $6.7 \times 10^{-8} \mathrm{~Pa}$. So, the minimum detectable partial pressure of MCQMS will reach a level of $<6.7 \times 10^{-8} \mathrm{~Pa}$. The sensitivity for this MCQMS is typically $1.5 \times 10^{-7} \mathrm{~A} / \mathrm{Pa}$, when operated at the normal electron emission current of $500 \mu \mathrm{A} /$ filament.

In order to further improve the sensitivity of the MCQMS, the resolution could be relaxed slightly. By ensuring that the peak width at $10 \%$ peak height is not wider than $1.0 \mathrm{amu}$, the sensitivity could be increased with a factor of 2 to 3 .

From the foregoing analysis it can be concluded that our MCQMS meets nicely our target specification. The most important objective has been realized: the MCQMS can be attached to vacuum devices as a measurement instrument and can be pumped simultaneously with them. Moreover, it can be sealed to small vacuum devices without affecting their performance significantly.

\section{PERFORMANCE COMPARISON BETWEEN MCQMS AND MINIATURE QMS}

The vacuum volume of the miniature QMS can be made very small by shrinking the quadrupole size. The effect of scaling down the radius and the length of the poles causes that sensitivity of the transmitted ions and the resolution will be decreased correspondingly. The following part contains a consideration on the design principle of the MCQMS with minimum volume, while maintaining a good resolution and sensitivity.

Sensitivity is an important parameter for a QMS. In order to discuss the sensitivity of QMS with different mechanical size, we assume that the ions injected into the orifice are uniform. Then, the product of the injection orifice $(S)$, the 


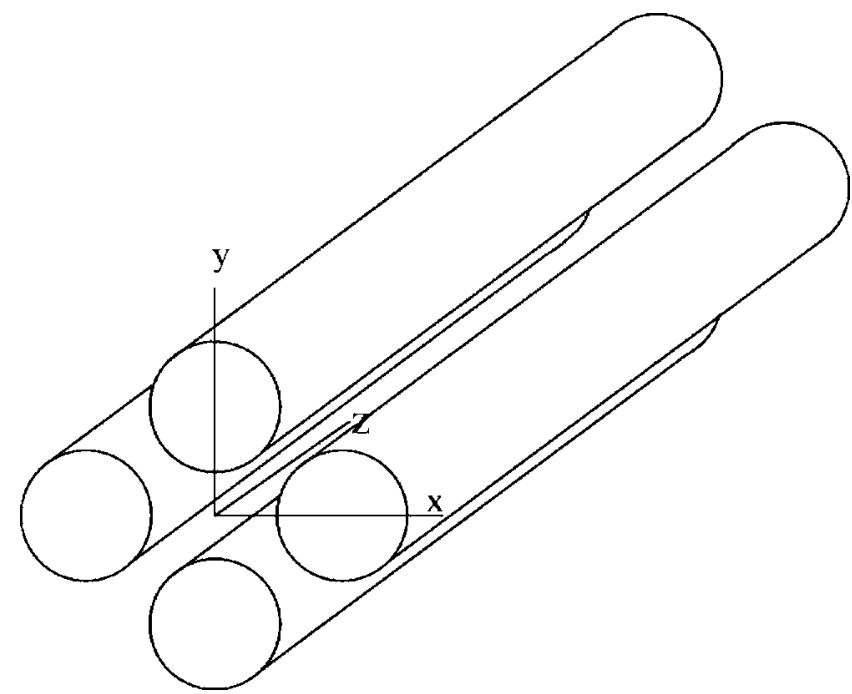

FIG. 6. Coordinates in the mass analyzing fiter.

ions' maximum allowable radial speed $\left(v_{r 0 m}\right)$, and the ions' maximum allowable axial energy $V_{m}$ can be used to describe the sensitivity approximatively as shown in Eq. (2):

Sensitivity $\propto S V_{m} v_{r 0 m}$.

To clarify the relationship between the QMS-size and the three parameters indicated in Eq. (2), a more in depth analysis will be presented in the following. This analysis is based on the work of Paul ${ }^{14}$ and Nagarajan. ${ }^{15}$

Figure 6 shows the coordinates in the mass analyzing system. Ions are injected into the mass filter and move along the $z$ axis. Only stable ions can be transmitted through the quadrupole field. The ion amplitude in the $x$ and $y$ directions should be smaller than the field radius $r_{0}$.

(1) We consider only ions, which are injected into the quadrupole field with an initial velocity parallel to the field axis. They can reach the detector only if the following conditions are satisfied:

$$
d=2 x_{0 m}=2 y_{0 m}=\frac{r_{0}}{(M / \Delta M)^{1 / 2}},
$$

where $x_{0 m}$ and $y_{0 m}$ are the maximum initial positions of the injected ions and $d$ is the diameter of the entrance orifice. From Eq. (3), it can be concluded that the scaling down of the quadrupole field size will lead to a quadratic reduction in the injection orifice.
(2) If ions enter from the ion source into the field of the mass filter, the transmission will depend on the initial axial velocity. The maximum allowable velocities for ion transmission $\dot{x}_{0 m}, \dot{y}_{0 m}$ are given by

$$
\dot{x}_{\text {om }}=0.32 r_{0}(M / \Delta M)^{1 / 2} \text {, }
$$

where a similar equation is valid for $\dot{y}_{0 m}$ From Eq. (4), it can be seen that the maximum allowable velocity is proportional to the field radius for a given resolution.

(3) The third important parameter for the injected ions is the energy in the direction of the field axis $\left(V_{z}\right)$. To achieve a better resolving power, $V_{z}$ should be in a certain range so that ions can make as many as possible oscillatory circles. However, reducing $V_{z}$ will reduce the efficiency of pulling ions out of the ionization chamber, so we need an optimization criterion.

If $V_{m}$ is the maximum allowable ion energy in the axial direction, then we can write

$$
V_{m}=4.2 \times 10^{2} f^{2} l^{2} M /(M / \Delta M),
$$

where $f$ is the frequency of the power supply in $\mathrm{MHz}$ and $l$ is the length of the $Q$-poles in $m$. From Eq. (5) is clear that $V_{m}$ is proportional to $l^{2}$ at the same frequency. In other words, by reducing the length of the QMS, the allowable energy has to be reduced even stronger. So, this condition will define the minimum length.

From Eqs. (3)-(5), the difference in sensitivity between the MCQMS and the miniature QMS can be calculated for ions of the same mass, at the same resolution and the same frequency of the QMS. Table I shows a comparison of the sensitivity between the MCQMS and miniature QMS. From this table we can see that the sensitivity of the MCQMS is 7982 times larger than that of a miniature QMS. To obtain a higher sensitivity, Ferran used a 14-18 MHz high frequency power supply and QMS-systems with 16 poles (9 groups) and 49 poles (36 groups) positioned parallel and reached a sensitivity of $10^{-6} \mathrm{~A} /$ Torr and consequently a detection limit of only $2 \times 10^{-9}$ Torr for partial pressures. ${ }^{16}$ The advantage of the miniature QMS is that it has a higher upper pressure limit because of its shorter ion trace.

Besides the difference in sensitivity, attention should also be paid to the resolving power $(M / \Delta M)$, which mainly depends on the geometrical precision of the quadrupole field, i.e., $r_{0} / \Delta r_{0}$. It is easy to understand that the geometrical pre-

\begin{tabular}{|c|c|c|c|c|c|c|c|}
\hline & $\begin{array}{c}\text { Pole diameter } \Phi \\
(\mathrm{mm})\end{array}$ & $\begin{array}{l}\text { Field radius } r_{0} \\
(\mathrm{~mm})\end{array}$ & $\begin{array}{l}\text { Pole length } l \\
\quad(\mathrm{~mm})\end{array}$ & Injection orifice ratio & $\begin{array}{c}\text { Allowable axial } \\
\text { velocity ratio }\end{array}$ & $\begin{array}{c}\text { Allowable ion } \\
\text { energy ratio }\end{array}$ & $\begin{array}{l}\text { Sensitivity } \\
\text { ratio }\end{array}$ \\
\hline MCQMS & & 3 & 100 & 46.5 & 6.82 & 25 & 7928 \\
\hline Miniature QMS & 1 & $0.44^{\mathrm{a}}$ & 20 & $1^{\mathrm{b}}$ & $1^{\mathrm{b}}$ & $1^{\mathrm{b}}$ & $1^{\mathrm{b}}$ \\
\hline
\end{tabular}
cision of the MCQMS quadrupole field can be much better

TABLE I. Comparison between the MCQMS and miniature QMS.

${ }^{\mathrm{a} C a l c u l a t e d ~ f r o m ~ E q . ~(1) . ~}$

${ }^{\mathrm{b}}$ Normalized. 
than that of a miniature quadrupole field: that is to say that the mass range of the miniature QMS will be limited by the precision of making the quadrupole field.

The stability of the sensitivity of a QMS is another crucial application parameter. The degradation of the sensitivity is caused by pollution of the surfaces of the quadrupole rods. During operation, electrons interact with the hydrocarbon gases in the vacuum chamber. This interaction leads to decomposition of these gases and forms carbonaceous deposits. So, during operation, the quadrupole's entrance surface may be covered with an insulation film. Charge accumulation will cause rf-field distortion at the entrance. For quadrupoles with a diameter of $6 \mathrm{~mm}$ and length of $50 \mathrm{~mm}$, both the sensitivity and mass spectra deteriorate after $\sim 100 \mathrm{~h}$ of operation at $1 \times 10^{-2} \mathrm{~Pa}$ in a normal vacuum system; particularly for gases with a low partial pressure, the measured peak height will be incorrect. Pollution becomes more serious when the quadrupoles get smaller or the ion current gets higher.

Since our MCQMS can be baked up to $500{ }^{\circ} \mathrm{C}$, these carbonaceous layers can largely be removed and allow a long operational period before cleaning the filter. Furthermore, the cleaning of the ceramic filter is easier than cleaning a manifold of micro poles.

\section{CONCLUSION}

We have designed a micro chamber QMS of which the mass filter is made of a ceramic body. The functional dimensions of the mass filter are well controlled and allow accurate residual gas analyses of vacuum devices with very small volumes. The internal volume of the MCQMS is less than $20 \mathrm{~cm}^{3}$ and the minimum detectable partial pressure is $10^{-8} \mathrm{~Pa}$ with a Faraday cup detector.

The robust design based on vacuum compatible ceramics enables high temperature baking and easy cleaning and maintenance after operation at high pressures. The resolution (at $10 \%$ peak height) in the mass range to $60 \mathrm{amu}$ is less than 1 amu. The MCQMS can be attached to small vacuum devices as a measurement instrument and can be pumped simultaneously with them.

\section{ACKNOWLEDGMENTS}

The project is supported by National Key Basic Research Program 973 (Grant Nos. 2003CB314702 and 2003CB314706), the Chinese 111 Project (Grant No. B07027), and Program for New Century Excellent Talents in University (Grant Nos. NCET-04-0473 and NCET-05-0466).

${ }^{1}$ R. J. Cotter, C. Fancher, and T. J. Cornish, J. Mass Spectrom. 34, 1368 (1999).

${ }^{2}$ Z. Ouyang, G. Wu, Y. Song, H. Li, W. R. Plass, and R. G. Cooks, Anal. Chem. 76, 4595 (2004).

${ }^{3}$ M. P. Sinha and A. D. Tomassian, Rev. Sci. Instrum. 62, 2618 (1991).

${ }^{4}$ C. B. Freidhoff et al., J. Vac. Sci. Technol. A 17, 2300 (1999).

${ }^{5}$ R. J. Cotter, C. Fancher, and T. J. Cornish, J. Mass Spectrom. 34, 1368 (1999).

${ }^{6}$ E. R. Badman and R. G. Cooks, J. Mass Spectrom. 35, 659 (2000).

${ }^{7}$ R. J. Ferran, U. S. Patent No. 5,401,962 (08/076,161 1995).

${ }^{8}$ S. Boumsellek and R. J. Ferran, J. Am. Soc. Mass Spectrom. 12, 633 (2001).

${ }^{9}$ R. J. Ferran and S. Boumsellek, J. Vac. Sci. Technol. A 14, 1258 (1996).

${ }^{10}$ S. Taylor et al., Vacuum 53, 203 (1999).

${ }^{11}$ J. J. Tunstall, S. Taylor, A. Vourdas, J. H. Leck, and J. Batey, Vacuum 53, 211 (1999).

${ }^{12}$ J. Jiao, A. J. Carella, G. S. Steeno, and R. T. Darrington, Int. J. Mass Spectrom. 216, 209 (2002).

${ }^{13}$ J. H. Batey, Vacuum 37, 659 (1987)

${ }^{14}$ W. Paul, H. P. Reinhard, and U. Von Zahn, Z. Phys. 152, 143 (1958).

${ }^{15}$ R. Nagarajan and P. K. Ghosh, J. Mass Spectrom. Ion Phys. 14, 267 (1974).

${ }^{16}$ D. H. Holkeboer, T. L. Karandy, F. C. Currier, L. C. Frees, and R. E. Ellefson, J. Vac. Sci. Technol. A 14, 3 (1996). 